\title{
Loss of splicing factor ASF/SF2 induces G2 cell cycle arrest and apoptosis, but inhibits internucleosomal DNA fragmentation
}

\author{
Xialu Li, Jin Wang, ${ }^{1}$ and James L. Manley ${ }^{2}$ \\ Department of Biological Sciences, Columbia University, New York, New York 10027, USA
}

\begin{abstract}
ASF/SF2 is an SR protein splicing factor that participates in constitutive and alternative pre-mRNA splicing and is essential for cell viability. Using a genetically modified chicken B-cell line, DT40-ASF, we now show that ASF/SF2 inactivation results in a G2-phase cell cycle arrest and subsequent programmed cell death. However, although several hallmarks of apoptosis are apparent, internucleosomal DNA fragmentation was not detected. Furthermore, inactivation of ASF/SF2 also blocks DNA fragmentation normally induced by a variety of apoptotic stimuli. Notably, mRNA encoding the inhibitor of caspase-activated DNase-L (ICAD-L), which acts as an inhibitor as well as a chaperone of caspase-activated DNase (CAD), decreased in abundance, whereas the level of mRNA encoding ICAD-S, which has only inhibitory activity, increased upon ASF/SF2 depletion. Strikingly, expression of appropriate levels of exogenous human ICAD-L restored apoptotic DNA laddering in ASF/SF2-depleted cells. These results not only indicate that loss of an SR protein splicing factor can induce cell cycle arrest and apoptosis, but also illustrate the important role of ICAD and its regulation by alternative splicing in the process of apoptotic DNA fragmentation.
\end{abstract}

[Keywords: ASF/SF2; splicing; apoptosis; ICAD; internucleosomal DNA cleavage]

Received July 26, 2005; revised version accepted September 9, 2005.

Most eukaryotic mRNAs are transcribed as precursors containing noncoding intervening sequences (introns), which are removed by splicing to form the mature mRNA. Recent analyses of the human genome have indicated that alternative splicing is a major source for a large proportion of the disparity between the modest number of genes in the human genome and the much higher complexity of the expressed proteome (Schmutz et al. 2004). Intron removal is catalyzed by a mechanism involving RNA cis-elements and their interaction with a complex repertoire of splicing factors, including snRNPs and non-snRNP proteins (Black 2003). The serine/arginine-rich (SR) protein family is one of the best-studied classes of splicing regulators. SR proteins are involved in multiple steps in the splicing reaction, including U1 snRNP binding to the 5' splice site, U2 snRNP binding to the branchpoint sequence at the $3^{\prime}$ splice site, U1-U2 interactions across the intron or exon, and joining of U4/ 5/6 tri-snRNP to the prespliceosome (Manley and Tacke

${ }^{1}$ Present address: Department of Biochemistry and Biophysics, University of Pennsylvania, Philadelphia, PA 19104.

${ }^{2}$ Corresponding author.

E-MAIL jlm2@columbia.edu; FAX (212) 865-8246.

Article published online ahead of print. Article and publication date are at http://www.genesdev.org/cgi/doi/10.1101/gad.1359305.
1996; Sanford et al. 2003). SR proteins are also capable of influencing splice site selection, largely by binding to regulatory elements in pre-mRNA and recruiting the general splicing machinery to the splicing signals. In contrast to their redundant roles in general splicing, individual SR proteins show diverse RNA binding specificities (Tacke and Manley 1999). Consistent with this, many naturally occurring splicing regulatory elements have been shown to bind specific SR proteins (e.g., see Caputi et al. 2004).

While the functions of SR proteins in splicing have been well documented in vitro, elucidation of their roles in vivo has been more difficult. Gene targeting experiments showed that SR proteins are essential for cell viability and/or animal development. In Caenorhabditis elegans, the siRNA-induced loss of the SR protein ASF/ SF2 or the simultaneous depletion of certain combinations of SR proteins caused developmental defects or lethality (Longman et al. 2000). In Drosophila, null alleles of the gene encoding B52/SRp55, another SR protein, resulted in lethality (Ring and Lis 1994). In vertebrates, genetic inactivation of ASF/SF2 in chicken DT40 cells was shown to result in a reduced rate of splicing and eventually cell death (Wang et al. 1996). Although changes in alternative splicing of reporter transcripts 
were observed, specific endogenous targets have not been identified (Wang et al. 1996; Lemaire et al. 2002). Knockout of the gene encoding SRp20 in the mouse blocked development at a very early stage (Jumaa et al. 1999). Conditional deletion of SC35 and ASF/SF2 in the mouse thymus and/or heart resulted in tissue-specific defects and changes in splicing of possible target transcripts (Wang et al. 2001; Ding et al. 2004; Xu et al. 2005). These studies all strongly support the view that specific SR proteins play critical functions important for cell viability and/or development. However, the specific role(s) that individual SR proteins play in general cell physiology is largely unknown.

One important cellular process in which alternative splicing and SR proteins likely play significant roles is programmed cell death or apoptosis. A remarkable number of transcripts encoding proteins that function in the apoptotic pathway are subject to alternative splicing such that the resultant proteins have opposite functions, either pro- or anti-apoptotic (Shin and Manley 2004; Schwerk and Schulze-Osthoff 2005). Although whether or not alternative splicing plays a significant physiological role in modulating apoptosis is not known, several studies have suggested that SR proteins may help to control splicing of pre-mRNAs encoding apoptotic regulators. For example, overexpression of SC35 was shown to alter splicing of mRNA encoding caspase- 2 such that increased levels of a pro-apoptotic form were produced, and this also enhanced cell death (Jiang et al. 1998). Changes in SR protein phosphorylation, which has the potential to influence alternative splicing (Cao et al. 1997; Xiao and Manley 1997, 1998; Prasad et al. 1999), have also been observed following induction of apoptosis by activation of the Fas receptor (e.g., see Utz et al. 1998). Nevertheless, whether changes in alternative splicing can regulate specific steps in the apoptotic pathway and whether individual SR proteins are necessary to modulate alternative splicing of mRNAs encoding apoptotic factors are not known.

Here we describe experiments initially aimed at characterizing how DT40 cells depleted of ASF/SF2 die. We show first that depletion causes a specific cell cycle arrest in G2 phase. Cell death subsequently ensues, following a pathway that displays many of the hallmarks of apoptosis. However, notable in its absence was any indication of internucleosomal cleavage or DNA laddering. Indeed, DNA laddering naturally brought about by classical apoptosis inducers was specifically inhibited by transient ASF/SF2 depletion. We show, by RNA analysis and transgenic rescue experiments, that this reflects a change in the alternative splicing of inhibitor of caspaseactivated DNase (ICAD) pre-mRNA, known to encode a regulator of the DNAse responsible for DNA laddering (Liu et al. 1997; Sakahira et al. 1998). Our findings establish that alternative splicing can indeed influence a specific step in the apoptotic pathway and that an SR protein is required for this regulation. Our results also provide evidence that ICAD plays a physiologically complex, significant role in regulating internucleosomal DNA cleavage.

\section{Results}

In vivo depletion of ASF/SF2 induces cell cycle arrest and apoptotic cell death

We previously described a derivative of chicken DT40 cells, DT40-ASF, in which the only copy of the ASF/SF2 gene is a human cDNA under the control of a tetracycline (tet)-repressible promoter (Wang et al. 1996). In the absence of tet, the growth properties and ASF/SF2 levels of these cells were indistinguishable from parental cells (Wang et al. 1996; data not shown). However, in the presence of tet, ASF/SF2 became undetectable by Western blot after 36-48 h, and cells stopped growing and began to die between 48 and $72 \mathrm{~h}$ after tet addition.

To gain more insight into the nature of these events, we first performed FACS analysis to determine the cell cycle profile of DT40-ASF cells incubated with tet for different periods of time (Fig. 1A). After $48 \mathrm{~h}$ of tet treatment, a noticeable increase of cells in G2/M phase $(4 \mathrm{~N}$ peak) was seen. After $72 \mathrm{~h}$, a large fraction of the cells $(45 \%)$ accumulated in G2/M phase, compared with only $9 \%$ of the untreated cells. Tet also did not affect the cell cycle distribution of parental DT40 cells /data not shown). To determine whether the cells were arrested in G2 or M phase, we examined the phosphorylation status of cdc2 kinase by Western blot (Fig. 1B). While cdc2 protein levels remain constant throughout the cell cycle, cdc2 activity is regulated by phosphorylation and de-

A

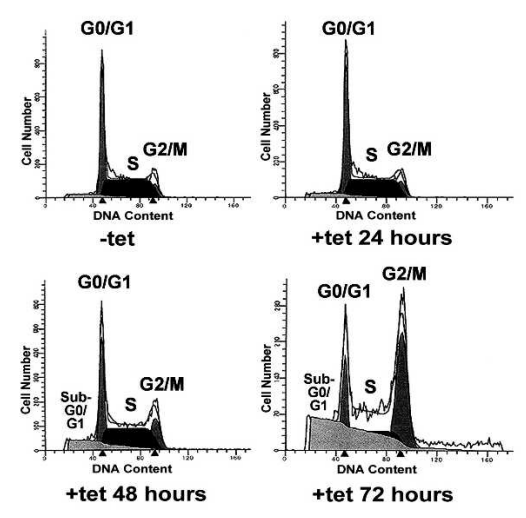

B

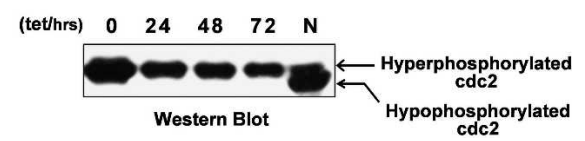

Figure 1. ASF/SF2 depletion induces G2-phase cell cycle arrest. (A) Cell cycle profiles of ASF/SF2-depleted cells. DT40-ASF cells treated with tet for $0,24,48$, or $72 \mathrm{~h}$ were stained with PI and analyzed by FACS. Raw data were quantitated by the ModFit program (Becton-Dickinson). Sub-G1, G0/G1, S, and G2/M phases are indicated. $(B)$ Western blot analysis was performed using whole-cell lysates from DT40-ASF cells treated with tet for $0,24,48$, or $72 \mathrm{~h}$ with anti-cdc2 antibodies. (N) Sample from nocodazol-synchronized cells. Positions of hypo- and hyperphosphorylated cdc2 are indicated. 
phosphorylation. At the G2/M boundary, cdc2 is activated by cdc25-mediated dephosphorylation and triggers entry into mitosis (for review, see Nurse 1990). As expected, cells synchronized with nocodazol, which are arrested in mitosis, contained a high level of hypophosphorylated cdc2. In contrast, no significant dephosphorylation of cdc2 was observed in ASF/SF2-depleted cells. These data indicate that ASF/SF2 is necessary, directly or indirectly, for G2-M progression, in a manner influencing cdc2 kinase activation. In addition, appearance of mitotic chromosomes was investigated by DAPI staining. No increase in the number of cells containing condensed mitotic chromosomes was detected in cells treated with tet for up to $72 \mathrm{~h}$ (data not shown). Significantly, comparable to what we observed in DT40-ASF cells, HeLa cells transfected with ASF/SF2 siRNA, but not a control siRNA, showed a striking increase in a cell population at G2-M phase concomitant with depletion of ASF/SF2, suggesting that the influence of ASF/SF2 on cell cycle progression is not limited to DT40 cells (Li and Manley 2005; data not shown). Taken together, these results indicate that $\mathrm{ASF} / \mathrm{SF} 2$ depletion induces G2phase cell cycle arrest.

In addition to G2 arrest, a significant increase in the number of sub-G0/G1 (<2N DNA content) cells was detected after ASF/SF2 depletion (Fig. 1A, 48 and 72 h; data not shown). Accumulation of sub-G0/G1 cells is a characteristic of cells undergoing apoptosis and reflects fragmentation of chromosomal DNA in such cells (Wyllie et al. 1980). To extend this result, we performed a TdTmediated dUTP nick-end labeling (TUNEL) assay (Fig. 2). A significant percentage of cells treated with tet for $72 \mathrm{~h}$ were labeled with fluorescence-conjugated dUTP in subG0/G1, G0/G1, and G2 phases, but very little in S phase, indicating that these cells indeed contained DNA
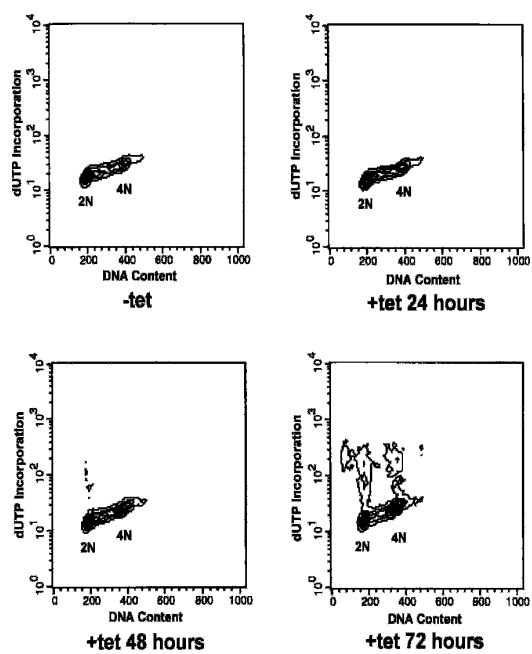

Figure 2. ASF/SF2 depletion induces TUNEL-positive cells. After tet treatment for 0, 24, 48, or $72 \mathrm{~h}$, cells were labeled with fluorescein-conjugated dUTP in the presence of TdT and subsequently stained with PI. The DNA content and incorporation of dUTP were measured simultaneously by FACS. G0/G1 (2N) and $\mathrm{G} 2 / \mathrm{M}(4 \mathrm{~N})$ phases are indicated.
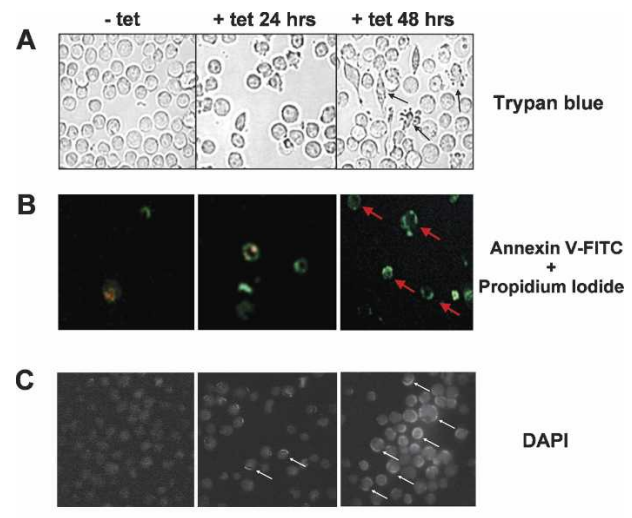

D

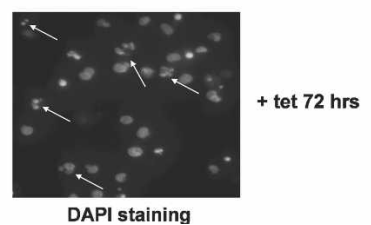

Figure 3. ASF/SF2 depletion induces apoptotic morphological changes. $(A-C)$ DT40-ASF cells treated with tet for 0,24 , or 48 $\mathrm{h}$ were stained with trypan blue $(A)$, Annexin V-FITC and PI $(B)$, or DAPI $(C)$. Arrows indicate typical apoptotic cells. $(D)$ Apoptotic bodies appear in DT40-ASF cells treated with tet for $72 \mathrm{~h}$. Arrows indicate typical apoptotic cells with fragmented nuclei.

breaks. The same cells were not labeled in a reaction lacking the TdT enzyme, nor were control DT40 cells grown in the presence or absence of tet (data not shown).

Loss of DNA integrity suggests but does not prove that ASF/SF2 depletion induces apoptotic death of DT40-ASF cells. To obtain additional evidence that cell death caused by ASF/SF2 depletion involved apoptosis, we tested whether the dying cells exhibited other characteristics of programmed cell death. First, we found that a significant fraction of ASF/SF2-depleted cells displayed typical surface morphological changes associated with apoptotic cell death, such as cytoplasmic shrinkage, membrane blebbing, and the appearance of apoptotic bodies (Fig. 3A). With tet treatment for up to $48 \mathrm{~h}$, the majority of DT40-ASF cells undergoing cell death were impermeable to trypan blue, indicating that membrane integrity was retained (Fig. 3A). Similarly, these cells were also resistant to the DNA-binding dye propidium iodide (PI), which cannot enter intact cells without permeabilization treatments because of its large size (Fig. 3B; data not shown). HeLa cells treated with ASF/SF2 siRNA behaved similarly (results not shown). Second, an Annexin V-positive and PI-negative stained cell population began to be observed at 48 -h tet treatment (Fig. 3B). Annexin $\mathrm{V}$ binding is a marker of phosphatidylserine (PS) externalization, a characteristic manifestation of apoptosis (van Engeland et al. 1998).

Nuclear morphology is the most accurate indicator of the involvement of apoptosis in cell death (Willingham 1999). We therefore next examined the nuclear morphology of ASF/SF2-depleted cells by DAPI staining. Treatment of cells with tet for as little as $24 \mathrm{~h}$ triggered chro- 
matin condensation against the nuclear periphery in some ASF/SF2-depleted cells. After 48 h, obvious nuclear rimming, known as stage I apoptotic chromatin condensation (chromatin condensed against the nuclear periphery), occurred in a significant fraction of ASF/SF2-depleted cells (Fig. 3C). Formation of discrete apoptotic bodies, stage II chromatin condensation, was also observed in $\sim 30 \%$ of cells treated with tet for $72 \mathrm{~h}$ (Fig. 3D; stage I and II chromatin condensation as defined by Susin et al. 1999; Daugas et al. 2000). Together, these results indicate that a significant fraction of ASF/SF2-depleted cells died by an apoptotic pathway.

\section{ASF/SF2 depletion influences apoptotic internucleosomal DNA fragmentation}

One of the best-studied biochemical characteristics of apoptosis is internucleosomal DNA cleavage, which generates a characteristic DNA ladder (Wyllie 1980; Earnshaw 1995). However, analysis of low molecular weight DNA by agarose gel electrophoresis failed to provide any evidence for DNA laddering in ASF/SF2-depleted DT40-ASF cells (Fig. 4A). One possible explanation for this is that DT40-ASF cells have one or more defects in the pathway resulting in apoptotic internucleosomal DNA cleavage. To test this, we first examined DNA ladder formation in staurosporine-treated DT40-ASF cells, with or without ASF/SF2 depletion. Staurosporine is a protein kinase inhibitor with a broad spectrum of activities (Ruegg and Burgess 1989), and can rapidly trigger both the morphological changes and internucleosomal DNA fragmentation typical of apoptosis in a variety of cell lines (Bertrand et al. 1994). Consistent with this, characteristic apoptotic DNA ladders were observed in DT40-ASF cells as early as $1.5 \mathrm{~h}$ after starurosporine treatment (Fig. 4B), indicating that DT40-ASF cells are capable of internucleosomal cleavage during apoptosis. Strikingly, no apoptotic DNA ladder was observed in DT40-ASF cells treated with tet for $48 \mathrm{~h}$, even after $3 \mathrm{~h}$ of starurosporine treatment (Fig. 4B). Staurosporine was still effective in inducing cell death, and resulted in similar changes in cell morphology in tettreated or untreated cells (data not shown). These data suggest that ASF/SF2 depletion was specifically responsible for the loss of internucleosomal DNA fragmentation in apoptotic DT40-ASF cells.

To extend the above results, we analyzed DNA ladder formation in DT40-ASF cells treated with diverse apoptotic stimuli at several time points following addition of tet (Fig. 4C). All of the apoptotic inducers caused DNA laddering in the absence of tet. However, in all cases, ASF/SF2 depletion inhibited internucleosomal DNA cleavage, such that by $48 \mathrm{~h}$ of tet treatment, the ability of all the compounds to induce DNA laddering was strongly inhibited. These results indicate that ASF/SF2 depletion causes a defect in internucleosomal DNA cleavage in apoptotic cells, pointing to an important role of ASF/SF2 in apoptotic DNA fragmentation.
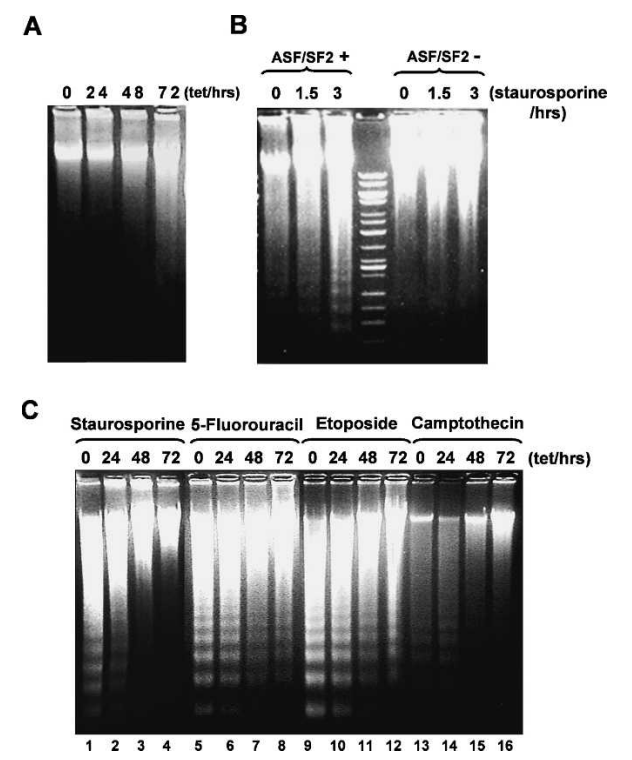

Figure 4. Internucleosomal DNA fragmentation is inhibited in ASF/SF2-depleted cells exposed to a variety of apoptotic stimuli. (A) Internucleosomal DNA cleavage in ASF/SF2-depleted cells. DT40-ASF cells were treated with or without 1 $\mu \mathrm{g} / \mathrm{mL}$ tet and harvested at the indicated time points. DNA fragmentation was assessed by $1.5 \%$ agarose gel electrophoresis. (B) Internucleosomal DNA fragmentation in ASF/SF2-depleted cells after exposure to $1.5 \mu \mathrm{M}$ staurosporine for different periods of time. DT40-ASF cells, with or without 2-d tet treatment, were exposed to $1.5 \mu \mathrm{M}$ staurosporine for $0,1.5$, or $3 \mathrm{~h}$ (as indicated). DNA fragmentation was assayed as above. $(C)$ Oligonucleosomal DNA fragmentation in ASF/SF2-depleted cells after $4 \mathrm{~h}$ exposure to apoptotic stimuli. After tet treatment for 0 , 24,48 , or $72 \mathrm{~h}$ (as indicated), DT40-ASF cells were exposed to $1.5 \mu \mathrm{M}$ staurosporine (lanes 1-4), $200 \mu \mathrm{M}$ 5-fluorouracil (lanes 5-8), $37.5 \mu \mathrm{M}$ etoposide (lanes 9-12), or $0.2 \mu \mathrm{M}$ camptothecin (lanes 13-16) for $4 \mathrm{~h}$. DNA fragmentation was assayed as above.

\section{ASF/SF2 depletion switches alternative splicing of ICAD pre-mRNA}

Considerable evidence indicates that DNA fragmentation factor, a heterodimeric protein complex consisting of the caspase-activated DNase (CAD/DFF40) and inhibitor of caspase-activated DNase (ICAD/DFF45/ CPAN), is largely responsible for internucleosomal DNA fragmentation during apoptosis (Liu et al. 1997, 1998; Enari et al. 1998; Sakahira et al. 1998). ICAD not only acts as an inhibitor of CAD, but also is required for the proper folding, formation and nuclear accumulation of a catalytically competent nuclease (Enari et al. 1998; Scholz et al. 2002). Significantly, in mouse and human cells, alternative splicing of ICAD pre-mRNA generates two mRNA isoforms, ICAD-S and ICAD-L, encoding proteins that exhibit similar inhibitory activity, but only one, ICAD- $\mathrm{L}$, is capable of providing the positive chaperone effects (Gu et al. 1999; Sakahira et al. 1998, 1999). These observations raise the possibility that the repressive effect of ASF/SF2 depletion on apoptotic DNA ladder formation was due to a switch in ICAD pre-mRNA alternative splicing. To test this, we first had to deter- 
mine the sequences of chicken ICAD mRNA isoforms and whether the mammalian pattern of alternative splicing occurs in DT40 cells.

In human and murine cells, the ICAD proteins are encoded by two mRNA isoforms generated by alternative splicing of intron 5 (Gu et al. 1999; Sakahira et al. 1999). In ICAD-L mRNA, intron 5 is removed by splicing, encoding a 331-amino-acid protein using a stop codon in exon 6 , while ICAD-S mRNA retains intron 5 and encodes a 268-residue isoform using a stop codon in intron 5. A group of chicken EST cDNAs highly similar to the C-terminal region of human ICAD-L was identified from BLAST database searches. The deduced amino acid sequences exhibit $58.5 \%$ identity to the human protein, indicating that the cloned gene represents the chicken homolog of ICAD-L. Using the chicken ICAD-L mRNA sequence, a genomic fragment was isolated from DT40 cells, containing sequences of exon 5 , intron 5 , and exon 6 (Fig. 5A). As in human and murine cells, two mRNAs with different 3 ' ends produced by alternative splicing of intron 5 were detected in DT40 cells (Fig. 5B; data not shown).

We were now in a position to investigate the possible role of ICAD pre-mRNA alternative splicing in the defective apoptotic DNA ladder formation in ASF/SF2-depleted cells. To this end, we analyzed the splicing pat-

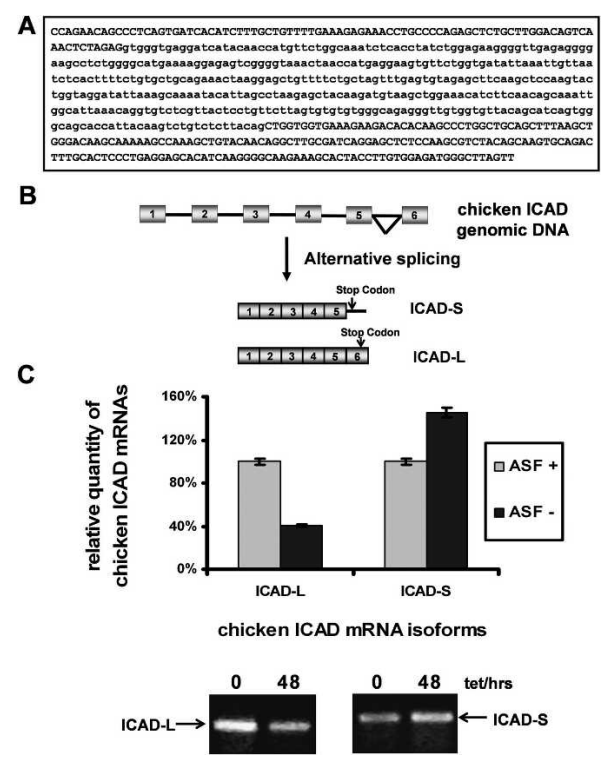

Figure 5. ASF/SF2 depletion switches alternative splicing of ICAD pre-mRNA. (A) Partial genomic sequence of chicken $I C A D-L$. Sequences of exon 5 and exon 6 are shown in uppercase, while the sequence of intron 5 is in lowercase. $(B)$ Scheme for alternative splicing pattern of chicken ICAD transcript that generates ICAD-L and ICAD-S mRNA isoforms. (C) Alternative splicing of ICAD pre-mRNA in DT40-ASF cells. RNA was isolated from DT40-ASF cells, with or without tet treatment for 48 $\mathrm{h}$, and ICAD transcripts quantitated by real-time RT-PCR (top panel) and standard RT-PCR (bottom panel). ICAD-L and ICAD-S mRNA levels were normalized to $\beta$-actin mRNA levels and the relative quantity of ICAD-L or ICAD-S mRNA is shown graphically. tern of ICAD pre-mRNA in DT40-ASF cells, untreated or treated with tet for $48 \mathrm{~h}$, by real-time and standard RTPCR. Compared with untreated cells, ICAD-L mRNA was decreased by $\sim 60 \%$, and ICAD-S mRNA was increased by $\sim 45 \%$ in the tet-treated cells, so that the ratio of ICAD-S to ICAD-L mRNA was increased by more than threefold (Fig. 5C). This change in ratio, although relatively small, was entirely reproducible, and was not seen in control DT40 cells treated with tet for $48 \mathrm{~h}$ /data not shown). Notably, depletion of ASF/SF2 by RNA interference strategy in HeLa cells also resulted in a twoto threefold similar decrease in the ratio of ICAD-L to ICAD-S proteins, reflecting a comparable switch on ICAD pre-mRNA alternative splicing and indicating that protein levels are also altered. These findings raised the possibility that the loss of internucleosomal DNA fragmentation brought about by ASF/SF2 depletion was caused by a change in the ratio of ICAD-L to ICAD-S.

\section{Exogenous human ICAD-L expression restores DNA ladder formation in ASF/SF2-depleted cells}

To provide evidence that the inhibitory effect of ASF/SF2 depletion on internucleosomal DNA cleavage is in fact mediated by the switch in ICAD pre-mRNA splicing, we set out to determine whether the loss of laddering could be recovered by stable expression of an appropriate level of exogenous ICAD-L. To this end, we transfected DT40ASF cells with a plasmid expressing human ICAD-L and isolated individual clones stably expressing different levels of ICAD-L, as judged by Western blot with anti-human ICAD antibodies (Fig. 6A). We then determined whether the pattern of DNA laddering resulting from 4-h treatment with etoposide was affected by hICAD-L, in the presence or absence of ASF/SF2 $( \pm 48$-h tet treatment) (Fig. 6B). Consistent with results described above (Fig. 4C), vector-transfected control cells displayed DNA laddering in the presence but not the absence of ASF/SF2 (Fig. 4C, lanes 1,2). In contrast, in cell lines expressing high levels of hICAD-L (\#69, 75, 77, and 80), DNA laddering was reduced in cells containing ASF/SF2 and remained undetectable in the ASF/SF2-depleted cells (Fig. 4C, lanes 9-16). These findings are consistent with previous overexpression studies indicating that high levels of ICAD-L inhibit CAD (Enari et al. 1998; Liu et al. 1998; Sakahira et al. 1998). Strikingly, however, cell lines expressing low levels of hICAD-L (\#62, 81, and 83) resulted in partial rescue of internucleosomal cleavage, as significant laddering was detected in the absence of ASF/SF2 (Fig. 4C, lanes 4,6,8). These results not only confirm the idea that correct levels of ICAD are essential for activation of CAD in vivo, but also, and more importantly, strongly support the idea that the reduced levels of ICAD-L brought about by the ASF/SF2 depletion-induced switch in alternative splicing is responsible for inhibition of apoptotic DNA laddering.

We next wished to determine whether DNA laddering could be induced by ASF/SF2 depletion alone in DT40ASF cells expressing low levels of exogenous ICAD-L. To this end, we treated DT40-ASF and the \#81 cells de- 
Li et al.

A

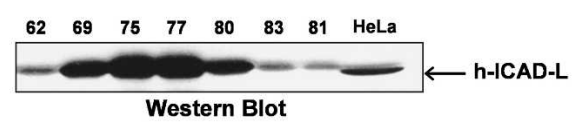

B

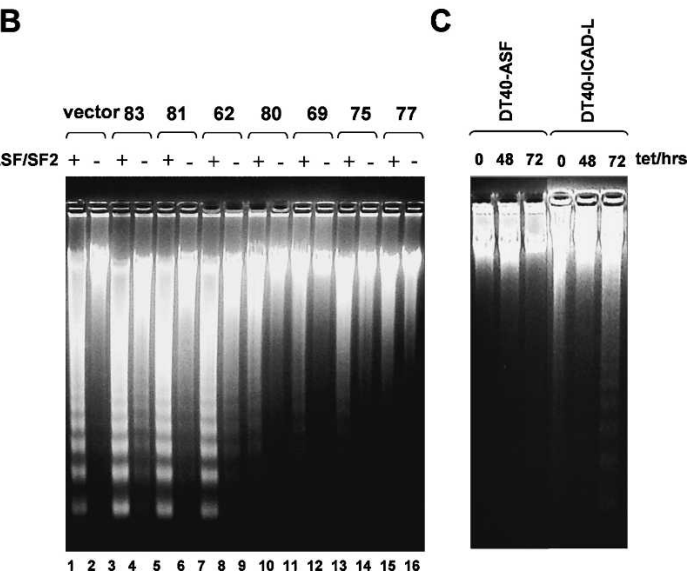

Figure 6. Proper levels of ICAD-L expression can partially restore apoptotic DNA ladder formation in ASF/SF2-depleted cells. (A) DT40-ASF cells were stably transfected with the human ICAD-L cDNA expression plasmid. Expression level of hICAD-L in stable transfectants (DT40-ICAD-L) was detected by Western blot with anti-ICAD-L antibody. HeLa cells were used as a control. The position of hICAD-L is indicated. $(B)$ Internucleosomal DNA fragmentation in DT40-ICAD-L cells after a 4-h exposure to etoposide, with or without ASF/SF2 depletion. Several DT40-ICAD-L colonies, with different levels of hICAD-L expression, were treated or not with $1 \mu \mathrm{g} / \mathrm{mL}$ tet for $48 \mathrm{~h}$ and exposed to $37.5 \mu \mathrm{M}$ etoposide for $4 \mathrm{~h}$. DNA ladder formation was assayed as above. $(C)$ Internucleosomal DNA fragmentation in ASF/SF2-depleted cells. DT40-ASF and DT40ICAD-L (\#81) cells were treated with tet for 0,48 , or $72 \mathrm{~h}$ (as indicated). Internucleosomal DNA fragmentation was assessed as above.

scribed above with tet and analyzed the pattern of DNA fragmentation (Fig. 6C). Strikingly, DNA ladders were detected in the ICAD-L expressing, but not the parental, DT40-ASF cells after ASF/SF2 depletion, providing additional evidence that the change in ICAD pre-mRNA splicing is responsible for the absence of internucleosomal DNA fragmentation following depletion of ASF/ SF2, and that an apoptotic pathway is in fact activated by loss of ASF/SF2.

\section{Discussion}

The data presented here indicate that loss of an SR protein splicing factor can lead to apoptotic cell death. We observed that genetic inactivation of ASF/SF2 resulted in cell cycle arrest in G2 phase and morphological changes associated with apoptosis, an exception being the lack of internucleosomal DNA fragmentation. This absence of DNA laddering reflects a change in alternative splicing of ICAD pre-mRNA resulting from ASF/SF2 depletion. Our data not only establish an association between ASF/ SF2, cell cycle progression, and apoptotic cell death, but also illustrate the physiological significance of the regulation of ICAD pre-mRNA alternative splicing in the process of apoptotic DNA fragmentation (Fig. 7A,B).

A number of genetic studies have revealed potential links between splicing and cell cycle progression. In both Saccharomyces cerevisiae and in Schizosaccharomyces pombe, a group of genes has been identified in independent screens for splicing factors and cell cycle regulators (Ben-Yehuda et al. 2000; Russell et al. 2000). In metazoans, disruption of $S A M 68$, which encodes an RNA-bind-
A

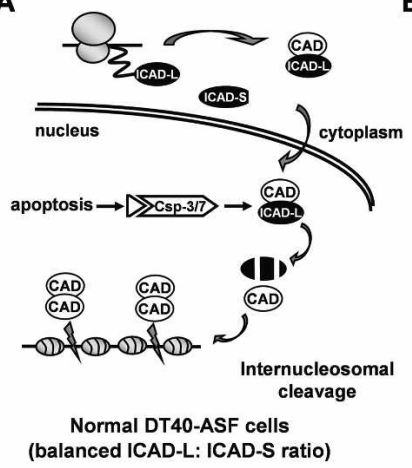

C

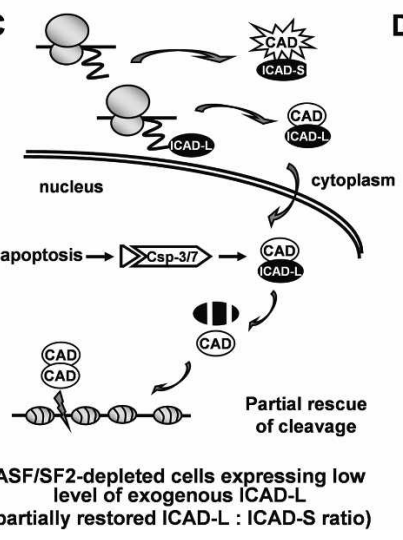

B

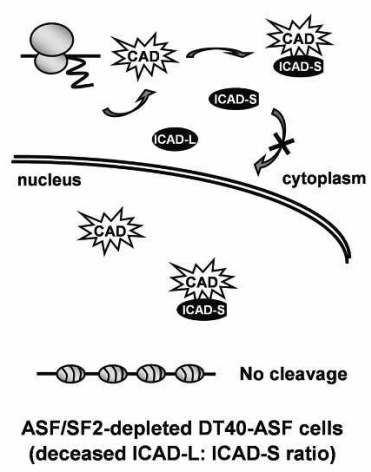

$D$

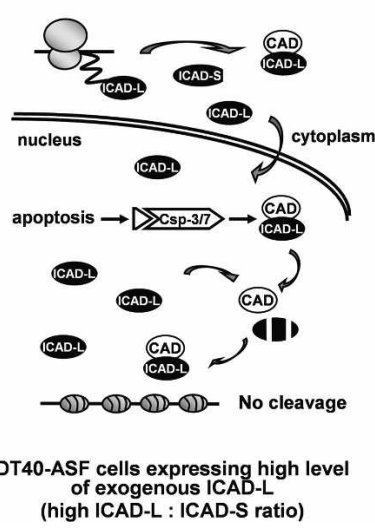

Figure 7. Control of apoptotic internucleosomal cleavage by alternative splicing. The diagrams depict how DNA fragmentation by CAD nuclease is modulated by shifts in the ratio of ICAD-L to ICAD-S isoforms. (A) ICAD-L (black oval), but not ICAD-S (black oval without right end), cotranslationally binds to nascent $\mathrm{CAD}$ and functions as a folding chaperone. ICAD-L remains bound to $\mathrm{CAD}$, keeping it in an inactive form, and facilitates the nuclear targeting of the heterodimeric complex. Properly folded CAD synthesized with ICAD-L is shown as a white oval, while inactive CAD synthesized without ICAD-L is shown as a multipoint star. Upon apoptotic stimuli, ICAD-L is proteolytically processed by caspase- 3 and caspase-7, releasing active $\mathrm{CAD}$ and initiating fragmentation of the chromosomal DNA. (B) Reduced levels of ASF/SF2 inhibit CAD-mediated cleavage by switching ICAD pre-mRNA splicing. Lowered ICAD-L levels prevent chaperoning, while elevated ICAD-S levels inhibit $\mathrm{CAD}$, in part by decreasing nuclear transport. $(C)$ Low levels of exogenous ICAD-L expression partially rescue DNA laddering in ASF/SF2-depleted cells, reflecting the partially restored ratio of ICAD-L to ICAD-S isoforms. $(D)$ High levels of exogenous ICAD-L expression inhibit DNA laddering even in the presence of ASF/SF2. 
ing protein with functions in pre-mRNA splicing, in DT40 cells resulted in retardation of G2-M-phase progression, but in a manner independent of cyclin/cdc2 kinase activity (Li et al. 2002). The exact molecular mechanism underlying most of these cases is still unclear. However, Dahan and Kupiec (2004) showed that CDC40/Prp17, a protein implicated in connecting the cell cycle and splicing in yeast, controls cell cycle progression via its role in splicing of a specific transcript, ANC1, which encodes a cell cycle regulator. Likewise, a $\mathrm{G} 2 / \mathrm{M}$ arrest caused by mutations in the splicing factor CDC5/Cef1 can be suppressed by removing the intron from a single target gene TUB1, encoding $\alpha$ tubulin (Burns et al. 2002). These results indicate that specific splicing factors can be required for cell cycle progression by influencing splicing of specific transcripts.

No significant differences have been detected following ASF/SF2 depletion in the metabolism of any mRNAs encoding cell cycle regulators (e.g., see Lemaire et al. 2002). Therefore, we must consider the possibility that something other than an alteration in pre-mRNA splicing might be responsible for the defect in the G2-M transition observed upon the loss of ASF/SF2. Consistent with this possibility, we recently observed that depletion of ASF/SF2 results in the rapid appearance of DNA double-strand breaks (DSBs), reflecting defects in transcription-coupled RNA processing (Li and Manley 2005). It is possible that such DSBs activate a DNA damage checkpoint (Smits and Medema 2001) and in turn arrest the cell cycle in G2. Understanding the mechanism of ASF/SF2 depletion-generated DSBs will elucidate at least part of the role of ASF/SF2 in cell cycle progression.

We previously demonstrated that DT40-ASF cells stopped growing and began to die between 48 and $72 \mathrm{~h}$ after ASF/SF2 depletion (Wang et al. 1996). We have shown here that a fraction of the dying cells display a number of morphological changes associated with apoptotic cell death (Willingham 1999), including cytoplasmic shrinkage, membrane blebbing, PS exposure, chromatin condensation, and the appearance of apoptotic bodies. Although we did not observe a significant increase in the levels of activated caspase- 3 proteins after ASF/SF2 depletion, we have recently found that the general caspase inhibitor, zVAD-fmk, partially suppressed ASF/SF2 depletion-induced DNA fragmentation after 2-d tet treatment, indicating that caspase activation does occur after ASF/SF2 depletion (Li and Manley 2005; data not shown).

The mechanism for activation of apoptosis upon ASF/ SF2 depletion could be explained in two possible scenarios. First, given that many genes appear to utilize alternative splicing to encode proteins with opposite effects on apoptosis (Shin and Manley 2004; Schwerk and Schulze-Osthoff 2005), it is possible that ASF/SF2 regulates alternative splicing of transcripts encoding celldeath regulators, and that this might contribute to activation of apoptosis in ASF/SF2-depleted cells. Indeed, we found that ASF/SF2 depletion correlates with a small but significant switch (five- to sixfold) in alternative splicing of the pre-mRNA encoding the cell death factor Bcl-X (J.
Wang, T. Kashima, X. Li, and J.L. Manley, unpubl.). Significantly, this switch not only is in keeping with the known properties of ASF/SF2 in modulating alternative splicing (e.g., see Manley and Tacke 1996), but also leads to an increase in the ratio of $\mathrm{Bcl}-\mathrm{X}_{\mathrm{S}}$, a pro-apoptotic isoform, to Bcl- $\mathrm{X}_{\mathrm{L}}$, an apoptotic inhibitor (Boise et al. 1993). Furthermore, overexpression of $\mathrm{Bcl}-\mathrm{X}_{\mathrm{L}}$ delays, but does not prevent, cell death caused by ASF/SF2 depletion (J. Wang, T. Kashima, X. Li, and J. Manley, unpubl.). It is possible that similar changes in the splicing patterns of transcripts from other apoptotic genes, together with the Bcl-X switch, promote apoptosis following ASF/SF2 depletion. In a second scenario, our recent studies showed that ASF/SF2 depletion results in extensive DNA damage, which can, in turn, activate a variety of cellular responses, including apoptosis ( $\mathrm{Li}$ and Manley 2005; for review, see Norbury and Zhivotovsky 2004). On the other hand, such global genomic alterations have the potential to influence expression of a number of genes, and this could lead to cell death by different pathways. In this context, it is reasonable that only a fraction of cells displayed evidence of apoptosis upon ASF/SF2 depletion.

Our experiments have provided evidence that a relatively small shift in ICAD pre-mRNA splicing is sufficient to suppress apoptotic DNA fragmentation. This raises a number of questions. First, is it reasonable that such a small change in the levels of ICAD-L and ICAD-S could have such a significant effect? A number of factors suggest that it is. For example, our data demonstrate that low levels, but only low levels, of ICAD-L are sufficient to partially rescue DNA laddering following ASF/SF2 depletion (Fig. 7C). This indicates that the relative levels of the two isoforms are very finely balanced such that a small shift could have a significant effect. Additionally, differences in the properties of the two proteins may serve to amplify the effects of small changes in concentration. In vitro binding assays have shown that ICAD-S has a significantly higher affinity for CAD than does ICAD-L (Gu et al. 1999). Additionally, the C-terminal domain of ICAD-L, absent in ICAD-S, includes the nuclear localization signal (NLS) (Samejima and Earnshaw 2000). In transient expression assays, loss of the ICAD-L NLS has been shown to shift the localization of a cotransfected CAD derivative from exclusively nuclear to equally distributed throughout the cell (Scholz et al. 2002). Thus the inhibitory ICAD-S isoform not only binds CAD more tightly than does ICAD-L, but also lowers its concentration in the nucleus.

The biochemical properties of ICAD-L are consistent with the importance of maintaining it at low intracellular concentrations, which, in turn, favors a finely tuned balance with ICAD-S. Despite the higher affinity of ICAD-S for CAD, only ICAD-L can provide the chaperone activity required for proper folding and activation of the enzyme detected in vitro (Enari et al. 1998; Gu et al. 1999; Sakahira et al. 1999). Indeed, CAD does not even accumulate to detectable levels in $I C A D^{-/-}$mouse embryo fibroblasts unless ICAD-L, but not ICAD-S, is expressed (Nagase et al. 2003). However, overexpression experiments have provided evidence that elevated levels 
of ICAD-L are inhibitory and prevent CAD activation (Enari et al. 1998; Liu et al. 1998; Sakahira et al. 1998). Our results are significant because they confirm the physiological significance of both these findings: Low levels of ICAD-L expression rescued DNA laddering in ASF/SF2-depleted cells, reflecting the reduced levels of ICAD-L in the depleted cells (Fig. 7C), but high levels resulted in inhibition of laddering even in the presence of ASF/SF2 (Fig. 7D). This complex mechanism underlying ICAD function likely contributes to the tight control of the CAD nuclease necessary to help ensure against inappropriate activation of the enzyme, and highlights the significance of the interplay between ICAD-L and ICAD-S.

How mechanistically does ASF/SF2 modulate ICAD pre-mRNA splicing? SR proteins generally function positively in splicing, so reduction in ASF/SF2 activity is consistent with the observed effect on ICAD splicing; that is, intron retention. However, SR protein function in most instances is largely redundant (for review, see Manley and Tacke 1996), and consistent with this, previous studies have found that genetic inactivation of ASF/SF2 has at most limited effect on splicing of most mRNAs (Wang et al. 1998; Lemaire et al. 2002; Xu et al. 2005). Why, then, is ICAD splicing affected by loss of ASF/SF2? A likely explanation is that exon 6 contains a sequence that closely resembles the high affinity A/Grich ASF/SF2-binding consensus determined by SELEX (Tacke and Manley 1995). Such sequences are capable of enhancing splicing of upstream introns when properly positioned in a downstream exon (Tacke and Manley 1995), and indeed a recent global analysis of alternative splicing events affected by siRNA-mediated depletion of the Drosophila homolog of ASF/SF2 revealed an overrepresentation of the consensus binding site in downstream exons (Blanchette et al. 2005).

Our data have shown that a subtle shift in ICAD premRNA splicing has a profound effect on a specific step in the programmed cell death pathway: turning on or off the process of internucleosomal DNA fragmentation. This is significant for two reasons. First, as mentioned above, numerous genes encoding apoptotic regulators or effectors produce transcripts by alternative splicing that give rise to proteins with potentially opposite effects on apoptosis. But in most cases these properties have been determined by transfection and/or biochemical studies employing isolated cDNAs (for review, see Schwerk and Schulze-Osthoff 2005). Our data show that simply reducing the levels of a single SR protein can indeed result in altering both splicing and a specific step in apoptosis. In this regard, it is noteworthy that concentrations and/or phosphorylation status of SR proteins vary in different tissues and cell types, and during different cell stages; for example, apoptosis (e.g., see Thiede et al. 2001). Thus our results illustrate how in a physiological setting a change in alternative splicing can indeed influence the apoptotic pathway. A second conclusion is that the process of internucleosomal DNA fragmentation can be turned on or off during apoptosis. It has been known that in some instances cells undergo apoptosis without DNA ladder- ing (e.g., see Boe et al. 1991), and that a related earlier step in the pathway, high molecular weight DNA fragmentation, still occurs in the absence of CAD nuclease (Samejima et al. 2001). However, genetic studies of mice and flies lacking CAD indicate that defects in internucleosomal fragmentation result in activation of innate immunity and defects in thymic development (Mukae et al. 2002; Kawane et al. 2003). While the significance of internucleosomal fragmentation in other apoptotic pathways remains to be determined, our data have shown that it can be turned on or off by a simple shift in alternative splicing, supporting the view that regulated splicing is an important mechanism in programmed cell death.

\section{Materials and methods}

\section{Constructs and transfections}

The cDNA for the coding region of human ICAD-L was obtained by RT-PCR of total HeLa cell RNA. Human ICAD-L cDNA under the control of the chicken $\beta$-actin promoter was inserted into the expression vector pAPSV-zeo /Chen and Manley 2000), generating the construct hICAD-L-Zeo. DT40-ASF cells were tranfected with hICAD-L-Zeo as described previously (Wang et al. 1996) and selected in medium containing $400 \mu \mathrm{g} /$ $\mathrm{mL}$ Zeocin (Invitrogen). Drug-resistant clones (DT40-ICAD-L) were expanded and screened for human ICAD-L expression by Western blot using anti-hICAD-L antibody (Santa Cruz Biotechnology). Levels of ASF/SF2 in cells were monitored by Western blot using the anti-flu epitope monoclonal antibody HA-11 (Covance Research Products).

\section{Cloning of chicken ICAD-L cDNA and genomic DNA}

A group of ESTs (accession nos. AJ449993, AJ455312, AJ454385, AJ451408, AJ449459, AJ449235, AJ448844, AJ446766, AJ445400) were identified by BLAST searches of the chicken EST database with sequences from human ICAD-L cDNA. Primers derived from the EST sequences were subsequently used to amplify a partial chicken ICAD-L cDNA from DT40 cells. Based on the sequences of this cDNA, a genomic DNA fragment containing intron 5 of ICAD-L was amplified from DT40 genomic DNA with primers located in exon 5 and exon 6.

Cell cycle analysis and TUNEL assays

Cells $\left(2 \times 10^{6}\right.$ to $\left.4 \times 10^{6}\right)$ were subjected to FACS analysis as described (Wang et al. 1996). TUNEL assays (Gavrieli et al. 1992) were performed as suggested by the manufacturer (Promega). Briefly, $3 \times 10^{6}$ DT40-ASF cells with or without 2 -d tet treatment were harvested and washed with ice-cold PBS. After being fixed in $5 \mathrm{~mL} \mathrm{1 \%}$ methanol-free formaldehyde, cells were permeablized by incubation in $5 \mathrm{~mL} 70 \%$ ethanol at $-20^{\circ} \mathrm{C}$ overnight. Following washing with PBS, cells were incubated in reaction mixtures containing fluorescein dUTP and TdT enzyme at $37^{\circ} \mathrm{C}$ for $1 \mathrm{~h}$. Cells were washed, resuspended in $1 \mathrm{~mL}$ PBS containing $5 \mu \mathrm{g} / \mathrm{mL}$ PI and $250 \mu \mathrm{g} / \mathrm{mL}$ RNase A and analyzed using a FACS Calibur (Becton Dickinson).

\section{Analysis of apoptotic cells}

(1) Cell morphology: Cells in 6-well plates were treated with trypan blue (Invitrogen) for $1 \mathrm{~min}$ and then examined under a 20x objective. Representative fields were photographed. (2) Nuclear morphology: Cells were washed with PBS, fixed with $3.7 \%$ formaldehyde, and permeabilized with $0.2 \%$ Triton X-100 
for 5 min. After washing with PBS, cells were incubated with DAPI labeling solution ( $2 \mu \mathrm{g} / \mathrm{mL}$ in PBS) for $5 \mathrm{~min}$ and examined under a fluorescence microscope. (3) Annexin-V-FLUOS and PI double staining: Cells $\left(1 \times 10^{6}\right)$ were washed with PBS. Cell pellets were resuspended into $100 \mu \mathrm{L}$ of labeling solution (Annexin-V-FLUOS and proidium iodid mixture; Roche) and analyzed by fluorescence microscopy.

\section{Real-time RT-PCR}

Total cellular RNA from DT40-ASF cells untreated or treated with $1 \mu \mathrm{g} / \mathrm{mL}$ tet for $48 \mathrm{~h}$ was isolated as described (Wang et al. 1996). One microgram total RNA was used in $20 \mu \mathrm{L}$ reverse transcription reaction mixture $(50 \mathrm{mM}$ Tris- $\mathrm{HCl}$ at $\mathrm{pH} 8.3,75$ $\mathrm{mM} \mathrm{KCl}, 3 \mathrm{mM} \mathrm{MgCl}, 10 \mathrm{mM}$ DTT, 20 U RNasin [Promega], $200 \mathrm{U}$ M-MLV reverse transcriptase [GIBCO-BRL], $0.5 \mathrm{mM}$ dNTP, and 20 pmol random hexamer), which was carried out at $37^{\circ} \mathrm{C}$ for $1 \mathrm{~h}$. Primers used in PCR to amplify ICAD-L cDNA were located in exons 5 and 6. Primers to amplify chicken ICAD-S cDNA were located in exon 4 and intron 5. (Sequences of primers are available upon request.) PCR was performed on an ABI 7700 Sequence Detector (Applied Biosystems) using SYBR Green fluorescence. The $25-\mu \mathrm{L}$ PCR reactions were composed of $5 \mu \mathrm{L}$ of 1:5 diluted cDNA, $12.5 \mu \mathrm{L}$ of $2 \times$ SYBR Green mix (Applied Biosystems), $3 \mu \mathrm{L}$ of primer mix (100 nM final concentration), and $4.5 \mu \mathrm{L}$ of water. Amplification conditions were $95^{\circ} \mathrm{C}$ for $30 \mathrm{sec}, 60^{\circ} \mathrm{C}$ for $1 \mathrm{~min}, 40$ cycles. PCR products were analyzed using the Sequence Detector software. After setting the threshold to compare the fluorescence of samples at identical points along the logarithmic portion of the amplification curve, relative RNA concentrations were calculated.

\section{DNA fragmentation assay}

Cells $\left(2 \times 10^{6} / \mathrm{mL}\right)$, with or without ASF/SF2 depletion, were cultured in the absence or presence of staurosporine $(1.5 \mu \mathrm{M})$, etoposide $(37.5 \mu \mathrm{M})$, camptothecin $(0.2 \mu \mathrm{M})$, or 5 -fluorouracil $(200 \mu \mathrm{M})$ for $4 \mathrm{~h}$ at $37^{\circ} \mathrm{C}$. DNA was extracted from $2 \times 10^{6}$ cells by overnight incubation at $56^{\circ} \mathrm{C}$ in $0.2 \mathrm{~mL}$ of lysis buffer $(50$ $\mathrm{mM}$ Tris. $\mathrm{HCl}$ at $\mathrm{pH} 7.5,10 \mathrm{mM}$ EDTA, $150 \mathrm{mM} \mathrm{NaCl}, 50$ $\mu \mathrm{g} / \mathrm{mL}$ of freshly dissolved proteinase K). DNA was recovered by isopropanol precipitation, resuspended in TE with $100 \mu \mathrm{g} / \mathrm{mL}$ of RNaseA, analyzed on $1.5 \%$ agarose gels, and visualized by ethidium bromide staining (Liu et al. 1998).

\section{Acknowledgments}

We are grateful to S. Bush for comments on the manuscript, to I. Boluk for help preparing the manuscript, and to T. Kashima for valuable discussions. This work was supported by NIH grant R37 GM 48259.

\section{References}

Ben-Yehuda, S., Dix, I., Russell, C.S., McGarvey, M., Beggs, J.D., and Kupiec, M. 2000. Genetic and physical interactions between factors involved in both cell cycle progression and pre-mRNA splicing in Saccharomyces cerevisiae. Genetics 156: $1503-1517$.

Bertrand, R., Solary, E., O'Connor, P., Kohn, K.W., and Pommier, Y. 1994. Induction of a common pathway of apoptosis by staurosporine. Exp. Cell Res. 211: 314-321.

Black, D.L. 2003. Mechanisms of alternative pre-messenger RNA splicing. Annu. Rev. Biochem. 72: 291-336.

Blanchette, M., Green, R.E., Brenner, S.E., and Rio, D.C. 2005. Global analysis of positive and negative pre-mRNA splicing regulators in Drosophila. Genes \& Dev. 19: 1306-1314.

Boe, R., Gjertsen, B.T., Vintermyr, O.K., Houge, G., Lanotte, M., and Doskeland, S.O. 1991. The protein phosphatase inhibitor okadaic acid induces morphological changes typical of apoptosis in mammalian cells. Exp. Cell Res. 195: 237-246.

Boise, L.H., Gonzalez-Garcia, M., Postema, C.E., Ding, L., Lindsten, T., Turka, L.A., Mao, X., Nunez, G., and Thompson, C.B. 1993. bcl-x, a bcl-2-related gene that functions as a dominant regulator of apoptotic cell death. Cell 74: 597-608.

Burns, C.G., Ohi, R., Mehta, S., O'Toole, E.T., Winey, M., Clark, T.A., Sugnet, C.W., Ares Jr., M., and Gould, K.L. 2002. Removal of a single $\alpha$-tubulin gene intron suppresses cell cycle arrest phenotypes of splicing factor mutations in Saccharomyces cerevisiae. Mol. Cell. Biol. 22: 801-815.

Cao, W., Jamison, S.F., and Garcia-Blanco, M.A. 1997. Both phosphorylation and dephosphorylation of ASF/SF2 are required for pre-mRNA splicing in vitro. RNA 3: 1456-1467.

Caputi, M., Freund, M., Kammler, S., Asang, C., and Schaal, H. 2004. A bidirectional SF2/ASF- and SRp40-dependent splicing enhancer regulates human immunodeficiency virus type $1 \mathrm{rev}$, env, vpu, and nef gene expression. J. Virol. 78: 65176526.

Chen, Z. and Manley, J.L. 2000. Robust mRNA transcription in chicken DT40 cells depleted of TAF(II)31 suggests both functional degeneracy evolutionary divergence. Mol. Cell. Biol. 20: 5064-5076.

Dahan, O. and Kupiec, M. 2004. The Saccharomyces cerevisiae gene CDC40/PRP17 controls cell cycle progression through splicing of the ANC1 gene. Nucleic Acids Res. 32: 25292540.

Daugas, E., Susin, S.A., Zamzami, N., Ferri, K.F., Irinopoulou, T., Larochette, N., Prevost, M.-C., Leber, B., Andrews, D., Penninger, J., et al. 2000. Mitochondrio-nuclear translocation of AIF in apoptosis and necrosis. FASEB J. 14: 729-739.

Ding, J.H., Xu, X., Yang, D., Chu, P.H., Dalton, N.D., Ye, Z., Yeakley, J.M., Cheng, H., Xiao, R.P., Ross, J., et al. 2004. Dilated cardiomyopathy caused by tissue-specific ablation of SC35 in the heart. EMBO J. 23: 885-896.

Earnshaw, W.C. 1995. Nuclear changes in apoptosis. Curr. Opin. Cell Biol. 7: 337-343.

Enari, M., Sakahira, H., Yokoyama, H., Okawa, K., Iwamatsu, A., and Nagata, S. 1998. A caspase-activated DNase that degrades DNA during apoptosis, and its inhibitor ICAD. $\mathrm{Na}$ ture 391: 43-50.

Gavrieli, Y., Sherman, Y., and Ben-Sasson, S.A. 1992. Identification of programmed cell death in situ via specific labeling of nuclear DNA fragmentation. J. Cell Biol. 119: 493-501.

Gu, J., Dong, R.P., Zhang, C., McLaughlin, D.F., Wu, M.X., and Schlossman, S.F. 1999. Functional interaction of DFF35 and DFF45 with caspase-activated DNA fragmentation nuclease DFF40. J. Biol. Chem. 274: 20759-20762.

Jiang, Z.H., Zhang, W.J., Rao, Y., and Wu, J.Y. 1998. Regulation of Ich-1 pre-mRNA alternative splicing and apoptosis by mammalian splicing factors. Proc. Nat1. Acad. Sci. 95: 91559160.

Jumaa, H., Wei, G., and Nielsen, P.J. 1999. Blastocyst formation is blocked in mouse embryos lacking the splicing factor SRp20. Curr. Biol. 9: 899-902.

Kawane, K., Fukuyama, H., Yoshida, H., Nagase, H., Ohsawa, Y., Uchiyama, Y., Okada, K., Iida, T., and Nagata, S. 2003. Impaired thymic development in mouse embryos deficient in apoptotic DNA degradation. Nat. Immunol. 4: 138144.

Lemaire, R., Prasad, J., Kashima, T., Gustafson, J., Manley, J.L., and Lafyatis, R. 2002. Stability of a PKCI-1-related mRNA is controlled by the splicing factor ASF/SF2: A novel function 
for SR proteins. Genes \& Dev. 16: 594-607.

Li, X. and Manley, J.M. 2005. Inactivation of the SR protein splicing factor ASF/SF2 results in genomic instability. Cell 122: 365-378.

Li, Q.H., Haga, I., Shimizu, T., Itoh, M., Kurosaki, T., and Fujisawa, J. 2002. Retardation of the G2-M phase progression on gene disruption of RNA binding protein Sam68 in the DT40 cell line. FEBS Lett. 525: 145-150.

Liu, X., Zou, H., Slaughter, C., and Wang, X. 1997. DFF, a heterodimeric protein that functions downstream of caspase-3 to trigger DNA fragmentation during apoptosis. Cell 89: $175-184$.

Liu, X., Li, P., Widlak, P., Zou, H., Luo, X., Garrard, W.T., and Wang, X. 1998. The 40-kDa subunit of DNA fragmentation factor induces DNA fragmentation and chromatin condensation during apoptosis. Proc. Natl. Acad. Sci. 95: 84618466.

Longman, D., Johnstone, I.L., and Caceres, J.F. 2000. Functional characterization of SR and SR-related genes in Caenorhabditis elegans. EMBO J. 19: 1625-1637.

Manley, J.L. and Tacke, R. 1996. SR proteins and splicing control. Genes \& Dev. 10: 1569-1579.

Mukae, N., Yokoyama, H., Yokokura, T., Sakoyama, Y., and Nagata, S. 2002. Activation of the innate immunity in Drosophila by endogenous chromosomal DNA that escaped apoptotic degradation. Genes \& Dev. 16: 2662-2671.

Nagase, H., Fukuyama, H., Tanaka, M., Kawane, K., and Nagata, S. 2003. Mutually regulated expression of caspase-activated DNase and its inhibitor for apoptotic DNA fragmentation. Cell Death Differ. 10: 142-143.

Norbury, C.J. and Zhivotovsky, B. 2004. DNA damage-induced apoptosis. Oncogene 23: 2797-2808.

Nurse, P. 1990. Universal control mechanism regulating onset of M-phase. Nature 344: 503-508.

Prasad, J., Colwill, K., Pawson, T., and Manley, J.L. 1999. The protein kinase Clk/Sty directly modulates SR protein activity: Both hyper- and hypophosphorylation inhibit splicing. Mol. Cell. Biol. 19: 6991-7000.

Ring, H.Z. and Lis, J.T. 1994. The SR protein B52/SRp55 is essential for Drosophila development. Mol. Cell. Biol. 14: 7499-7506.

Ruegg, U.T. and Burgess, G.M. 1989. Staurosporine, K-252 and UCN-01: Potent but nonspecific inhibitors of protein kinases. Trends Pharmacol. Sci. 10: 218-220.

Russell, C.S., Ben-Yehuda, S., Dix, I., Kupiec, M., and Beggs, J.D. 2000. Functional analyses of interacting factors involved in both pre-mRNA splicing and cell cycle progression in Saccharomyces cerevisiae. RNA 6: 1565-1572.

Sakahira, H., Enari, M., and Nagata, S. 1998. Cleavage of CAD inhibitor in CAD activation and DNA degradation during apoptosis. Nature 391: 96-99.

- 1999. Functional differences of two forms of the inhibitor of caspase-activated DNase, ICAD-L, and ICAD-S. J. Biol. Chem. 274: 15740-15744.

Samejima, K. and Earnshaw, W.C. 2000. Differential localization of ICAD-L and ICAD-S in cells due to removal of a C-terminal NLS from ICAD-L by alternative splicing. Exp. Cell Res. 255: 314-320.

Samejima, K., Tone, S., and Earnshaw, W.C. 2001. CAD/DFF40 nuclease is dispensable for high molecular weight DNA cleavage and stage I chromatin condensation in apoptosis. J. Biol. Chem. 276: 45427-45432.

Sanford, J.R., Longman, D., and Caceres, J.F. 2003. Multiple roles of the SR protein family in splicing regulation. Prog. Mol. Subcell. Biol. 31: 33-58.

Schmutz, J., Wheeler, J., Grimwood, J., Dickson, M., Yang, J.,
Caoile, C., Bajorek, E., Black, S., Chan, Y.M., Denys, M., et al. 2004. Quality assessment of the human genome sequence. Nature 429: 365-368.

Scholz, S.R., Korn, C., Gimadutdinow, O., Knoblauch, M., Pingoud, A., and Meiss, G. 2002. The effect of ICAD-S on the formation and intracellular distribution of a nucleolytically active caspase-activated DNase. Nucleic Acids Res. 30: 3045-3051.

Schwerk, C. and Schulze-Osthoff, K. 2005. Regulation of apoptosis by alternative pre-mRNA splicing. Mol. Cell 19: 1-13.

Shin, C. and Manley, J.L. 2004. Cell signalling and the control of pre-mRNA splicing. Nat. Rev. Mol. Cell Biol. 5: 727-738.

Smits, V.A. and Medema, R.H. 2001. Checking out the G(2)/M transition. Biochim. Biophys. Acta 1519: 1-12.

Susin, S.A., Lorenzo, H.K., Zamzami, N., Marzo, I., Snow, B.E., Brothers, G.M., Mangion, J., Jacotot, E., Costantini, P., Loeffler, M., et al. 1999. Molecular characterization of mitochondrial apoptosis-inducing factor. Nature 397: 441-446.

Tacke, R. and Manley, J.L. 1995. The human splicing factors ASF/SF2 and SC35 possess distinct, functionally significant RNA binding specificities. EMBO J. 14: 3540-3551.

1999. Determinants of SR protein specificity. Curr. Opin. Cell Biol. 11: 358-362.

Thiede, B., Dimmler, C., Siejak, F., and Rudel, T. 2001. Predominant identification of RNA-binding proteins in Fas-induced apoptosis by proteome analysis. I. Biol. Chem. 276: 26044-26050.

Utz, P.J., Hottelet, M., van Venrooij, W.J., and Anderson, P. 1998. Association of phosphorylated serine/arginine (SR) splicing factors with the U1-small ribonucleoprotein (snRNP) autoantigen complex accompanies apoptotic cell death. J. Exp. Med. 187: 547-560.

van Engeland, M., Nieland, L.J., Ramaekers, F.C., Schutte, B., and Reutelingsperger, C.P. 1998. Annexin V-affinity assay: A review on an apoptosis detection system based on phosphatidylserine exposure. Cytometry 31: 1-9.

Wang, J., Takagaki, Y., and Manley, J.L. 1996. Targeted disruption of an essential vertebrate gene: ASF/SF2 is required for cell viability. Genes \& Dev. 10: 2588-2599.

Wang, J., Xiao, S.H., and Manley, J.L. 1998. Genetic analysis of the SR protein ASF/SF2: Interchangeability of RS domains and negative control of splicing. Genes \& Dev. 12: 22222233.

Wang, H.Y., Xu, X., Ding, J.H., Bermingham Jr., J.R., and Fu, X.D. 2001. SC35 plays a role in T cell development and alternative splicing of CD45. Mol. Cell 7: 331-342.

Willingham, M.C. 1999. Cytochemical methods for the detection of apoptosis. J. Histochem. Cytochem. 47: 11011110 .

Wyllie, A.H. 1980. Glucocorticoid-induced thymocyte apoptosis is associated with endogenous endonuclease activation. Nature 284: 555-556.

Wyllie, A.H., Kerr, J.F., and Currie, A.R. 1980. Cell death: The significance of apoptosis. Int. Rev. Cytol. 68: 251-306.

Xiao, S.H. and Manley, J.L. 1997. Phosphorylation of the ASF/ SF2 RS domain affects both protein-protein and proteinRNA interactions and is necessary for splicing. Genes \& Dev. 11: 334-344.

- 1998. Phosphorylation-dephosphorylation differentially affects activities of splicing factor ASF/SF2. EMBO $J$. 17: 6359-6367.

Xu, X., Yang, D., Ding, J.H., Wang, W., Chu, P.H., Dalton, N.D., Wang, H.Y., Bermingham Jr., J.R., Ye, Z., Liu, F., et al. 2005. ASF/SF2-regulated CaMKII $\delta$ alternative splicing temporally reprograms excitation-contraction coupling in cardiac muscle. Cell 120: 59-72. 


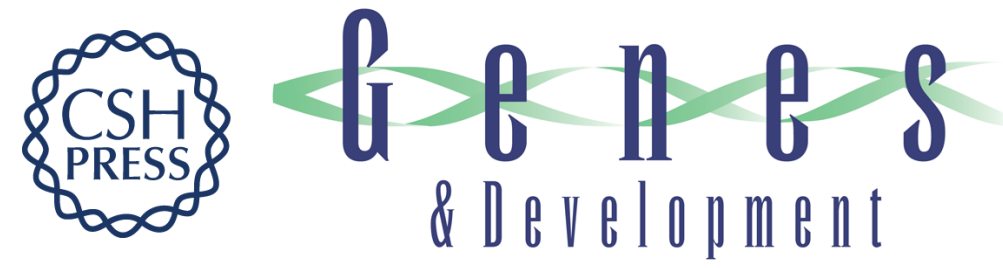

\section{Loss of splicing factor ASF/SF2 induces G2 cell cycle arrest and apoptosis, but inhibits internucleosomal DNA fragmentation}

Xialu Li, Jin Wang and James L. Manley

Genes Dev. 2005, 19:

Access the most recent version at doi:10.1101/gad.1359305

References This article cites 59 articles, 26 of which can be accessed free at: http://genesdev.cshlp.org/content/19/22/2705.full.html\#ref-list-1

License

Email Alerting

Receive free email alerts when new articles cite this article - sign up in the box at the top Service right corner of the article or click here.

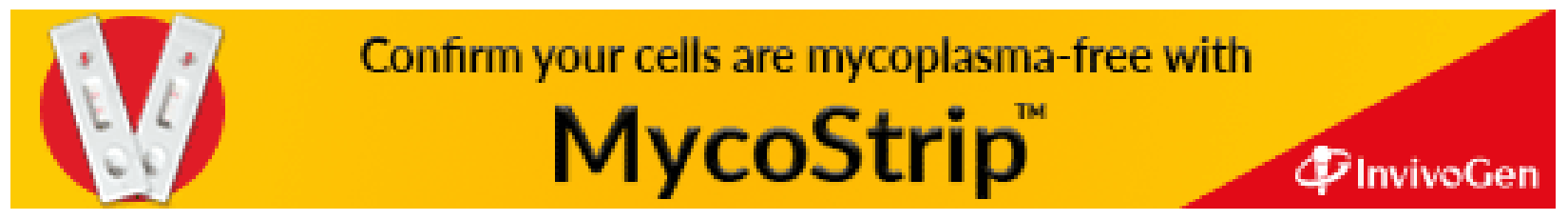

